\title{
Belgeo
}

Revue belge de géographie

$1 \mid 2008$

Aspects of the history of the Belgian geography and cartography

\section{Aspects of the history of the Belgian geography and cartography. Editorial}

\section{Henri Nicolaï}

\section{(2) OpenEdition}

\section{Journals}

Édition électronique

URL : http://journals.openedition.org/belgeo/10159

DOI : $10.4000 /$ belgeo.10159

ISSN : 2294-9135

Éditeur :

National Committee of Geography of Belgium, Société Royale Belge de Géographie

Édition imprimée

Date de publication : 31 mars 2008

Pagination : 3-4

ISSN : 1377-2368

Référence électronique

Henri Nicolaï, « Aspects of the history of the Belgian geography and cartography. Editorial », Belgeo [En ligne], 1 | 2008, mis en ligne le 19 octobre 2013, consulté le 22 septembre 2020. URL : http:// journals.openedition.org/belgeo/10159; DOI : https://doi.org/10.4000/belgeo.10159

Ce document a été généré automatiquement le 22 septembre 2020

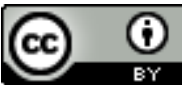

Belgeo est mis à disposition selon les termes de la licence Creative Commons Attribution 4.0 International. 


\title{
Aspects of the history of the Belgian geography and cartography. Editorial
}

\author{
Henri Nicolaï
}

1 Ce fascicule de Belgeo envisage quelques facettes de l'histoire de la géographie et de la cartographie en Belgique. Elles concernent des aspects apparemment mineurs mais qui ne sont pas sans intérêt. Il est question de géographie mais aussi et surtout de personnages ou de groupes qui ont cherché à mieux faire connaître la géographie et qui en même temps l'ont utilisée pour mener à bien leurs propres projets.

2 Les trois articles qui traitent directement de la géographie montrent en effet trois aspects différents de son utilisation.

3 Dans l'article de Jan Vandersmissen, on voit Charles Sainctelette, homme politique wallon, militer pour la création d'une société belge de géographie, à l'instar de celle qui avait été créée en France en 1821 et qui avait été suivie par les sociétés de Londres, de Vienne, de Berlin et de Saint-Pétersbourg. Il estime en effet que les habitants, et plus spécialement les milieux d'affaires d'un pays comme la Belgique, qui connaît à ce moment un grand essor industriel et économique, ont besoin d'informations correctes sur les autres pays du monde qui constituent un marché extérieur à prospecter, offrent des débouchés pour les produits nationaux et éventuellement fournissent des matières premières. La collecte et la diffusion de ces informations lui paraissent constituer le rôle primordial d'une société de géographie. Il voudrait d'ailleurs la localiser, non dans la capitale politique du pays, mais à Anvers, qui commence à s'affirmer à nouveau comme grande métropole commerciale. Mais ses mauvaises relations, dont certaines de nature politique, avec les géographes et les milieux d'affaires anversois, font échouer son entreprise. L'échec est quelque peu paradoxal puisqu'au même moment, les milieux anversois réussissent brillamment l'organisation du premier congrès international de Géographie. Mais peut-être les objectifs purement commerciaux de son projet n'avaient-ils pas été de nature à attirer suffisamment les milieux scientifiques. Les 
sociétés belges de géographie naîtront quelques années plus tard en plaçant davantage l'accent sur les préoccupations scientifiques et humanitaires.

4 L'article d'Elise Henry décrit comment A.-J. Wauters met la géographie au service du projet colonial de Léopold II. Le roi avait fait de la science géographique le ferment initial de son entreprise en réunissant en 1876 la Conférence géographique de Bruxelles en même temps qu'il exaltait son volet humanitaire (apport de la civilisation et lutte contre la traite et l'esclavage). Il avait encouragé tacitement la création de sociétés de géographie. En 1884, peu avant que son projet ne prenne sa forme politique - l'Etat Indépendant du Congo sera reconnu officiellement en 1885 - la création du Mouvement Géographique lui fournit un organe efficace de propagande. Le journal qui sera publié jusqu'en 1921 est une mine d'informations sur les opérations coloniales du dernier quart du XIX ${ }^{e}$ siècle en Afrique et dans le Monde mais surtout au Congo. Il a familiarisé l'opinion publique nationale à la participation des Belges à "l'oeuvre coloniale". Le journal a toujours protesté de son indépendance mais l'auteur montre qu'il a été soutenu directement par le roi, du moins à certains moments et surtout dans les premières années de son existence. L'auteur a trouvé, dans la correspondance d'un collaborateur du roi, la preuve du paiement du traitement du rédacteur en chef par le roi lui-même. Cela ne signifie pas qu'il y eut une intervention directe dans le contenu même du journal mais montre l'importance du rôle qu'on en attendait. Le journal devint par la suite l'organe des sociétés belges travaillant au Congo, se détacha de la politique économique de Léopold II et attaqua sa politique des monopoles.

C'est une illustration beaucoup plus consensuelle de la géographie qu'illustre l'article de Henri Nicolaï. Une commission créée par la Ville de Bruxelles, il y a exactement un siècle, a décidé d'initier les écoliers au sentiment du beau en exposant dans les locaux scolaires des estampes chromolithographiques représentant des paysages géographiques de la Belgique et commandées à des artistes réputés. Ces estampes donnent une bonne idée de la représentation géographique que l'on se faisait de la Belgique au début du $\mathrm{XX}^{\mathrm{e}}$ siècle, notamment par ses paysages traditionnels. Ceux-ci sont rendus fidèlement, avec le souci d'exprimer leurs caractéristiques, parfois même en les modélisant, voire, dans certains cas, en reflétant les appréciations et même les préjugés qu'on leur portait à l'époque. Cette utilisation de la géographie dans un but esthétique nous change des noirs desseins attribués parfois aux géographes.

Les articles consacrés à l'histoire de la cartographie apportent essentiellement des éléments nouveaux sur deux points : d'une part le degré de précision des cartes établies au XVI e siècle pour le territoire de la Flandre et d'autre part l'état de la cartographie de la Belgique, à la veille de son indépendance, pendant la période dite hollandaise.

7 F. Depuydt et al. ont cherché à mesurer la précision de cartes anciennes du comté de Flandre par rapport aux cartes actuelles et notamment en comparant les positions relatives d'éléments identifiables dans les deux cas comme certains bâtiments (églises par exemple) ou parties de villes ou de villages. Le traitement informatique des données et l'utilisation d'un type particulier d'analyse ont été effectués pour trois cartes du XVI e siècle, celle de G. Mercator (1540), celle figurant dans l'atlas d'A. Ortelius (1570) et celle du Brugse Vrije peinte par P. Pourbus (1571). Le degré de précision de la carte de Mercator est assez grand dans sa partie centrale, notamment dans le pays de Waas, et diminue sur les bords. La similitude des déviations mesurées sur cette carte et sur celle d'Ortelius confirme bien que cette dernière est une copie de la première à échelle réduite, ce que mentionne d'ailleurs Ortelius lui-même, avec l'addition d'une 
quarantaine de lieux (et, dans leurs cas, une moins bonne précision). L'analyse de la carte de Pourbus qui est à une échelle beaucoup plus grande, montre qu'elle n'est pas une copie de la carte de Mercator mais qu'elle a dû utiliser d'autres sources dont des triangulations relativement précises.

Lisette Danckaert, après avoir d'abord estimé la part de la géographie dans la bibliothèque d'un homme cultivé de la première moitié du XIX ${ }^{e}$ siècle et après avoir évoqué les cartes inspirées de Ferraris et mises à jour par un de ses anciens collaborateurs, rend compte des tentatives topographiques à moyenne et grande échelle, relativement peu connues,oeuvres d'auteurs indépendants, de 1815 à 1830 . Il s'agit de feuilles dressées par Mme Monborne couvrant la région frontalière avec la France ainsi que Bruxelles et ses environs. Pour les cartes des régions frontalières, Monborne semble avoir puisé nombre d'éléments dans les cartes hollandaises des Militaire Verkenningen (c'est-à-dire les Reconnaissances militaires), levées dans les années qui ont suivi la création du Royaume des Pays-Bas. Les feuilles de la région de Bruxelles sont comparées, dans leurs détails et dans les procédés techniques, à celles dressées au même moment par Guillaume de Wauthier pour le même territoire ainsi que pour les environs de Malines.

Une contribution de Bernard Jouret consacrée plus spécialement à l'oeuvre cartographique du grand personnage du XIX ${ }^{\mathrm{e}}$ siècle qu'a été Jean-Charles Houzeau, et notamment à sa réalisation d'une carte générale du relief de l'Europe, avec courbes de niveau, avait été initialement prévue pour ce fascicule. Comme elle a été présentée dans un colloque qui s'est tenu à la Bibliothèque Royale en 2007, elle figurera dans les actes de ce colloque qui seront publiés dans un prochain fascicule de BELGEO intitulé "Formatting Europe. Mapping a Continent".

Enfin une note, par Christian Vandermotten, s'efforce d'évaluer la place des "géographes" dans la mémoire collective belge en estimant la part que leur ont réservée les biographies nationales et les éléments que celles-ci en ont retenus pour les différentes périodes de l'histoire.

11 Ce fascicule comporte aussi - mais hors thème cette fois - un texte inédit de Pierre Gourou. Ecrit en 1986-1987, à un moment où foisonnent les livres consacrés à l'origine et à l'identité de la France, peu avant la commémoration du deux centième anniversaire de la Révolution, il exprime le point de vue de Pierre Gourou sur ces questions et sur la façon dont elles ont été formulées. Cet essai de géographie historique et politique illustre la diversité des thèmes qui retenaient son attention, audelà des thèmes de géographie tropicale qui ont fait sa réputation.

\section{AUTEUR}

\section{HENRI NICOLAÏ}

Université Libre de Bruxelles, henri.nicolai@scarlet.be 